\title{
Aprotinin: Twenty-five years of claim and counterclaim
}

\author{
Stephen Westaby, PhD, FECTS, FACC
}

See related articles on pages 495,573 , and 685 .
From the Oxford Heart Centre, John Radcliffe Hospital, Oxford, United Kingdom.

Received for publication Jan 14, 2008; accepted for publication Jan 14, 2008.

Address for reprints: Stephen Westaby, BSc, MS, PhD, Oxford Heart Centre, John Radcliffe Hospital, Oxford 0X9 3DU, United Kingdom (E-mail: swestaby@ahf. org.uk).

J Thorac Cardiovasc Surg 2008;135:487-91 $0022-5223 / \$ 34.00$

Copyright $(\underset{20}{ } 2008$ by The American Association for Thoracic Surgery

doi:10.1016/j.jtcvs.2008.01.002
7 he coagulopathy after blood-foreign surface interaction in the cardiopulmonary bypass circuit is part of the "postperfusion syndrome," which historically has accounted for as many deaths as has cardiac insufficiency. Cardiopulmonary bypass induces platelet dysfunction, thrombin production, and plasmin release. ${ }^{1}$ These changes provide the basis for postoperative hemorrhage, cardiac tamponade, and the need for surgical re-entry or excessive blood transfusion. Antiplatelet therapy in patients with coronary artery disease further compounded these effects. In turn, transfusion of packed red blood cells and coagulation components is recognized to increase the risk of morbidity and mortality after cardiac surgery. ${ }^{2,3}$

\section{An Important Discovery}

In the early 1980s, the Kirklin group in Alabama began to pursue the pathophysiologic mechanisms underlying the postperfusion syndrome. In short, blood-foreign surface interaction and protamine administration were both found to activate complement. Complement anaphylatoxins caused neutrophil activation, intrapulmonary white cell sequestration, and release of protease enzymes and free radicals. Twentyfive years ago at the Hammersmith Hospital, my colleagues and I sought to attenuate the inflammatory response by using the only commercially available protease inhibitor, aprotinin, which had been used unsuccessfully to treat pancreatitis. At this stage, no interaction was known or anticipated between aprotinin and the coagulation system. The hemostatic mechanism was discovered when the surgical field was noted to be abnormally dry after cardiopulmonary bypass. As the therapeutic potential became apparent, guidelines for aprotinin use were developed by trial and error. Using the standard celite-activated clotting time (ACT) of greater than 450 seconds for heparin monitoring, blood often set solid in the pericardium with a shiny glazed appearance. Intraoperative events such as spontaneous vein graft thrombosis or clotting within the oxygenator were not uncommon until it was recognized that aprotinin alone prolonged the celite $\mathrm{ACT}$ and the in vitro activated partial thromboplastin time. ${ }^{4}$ This has important implications for heparin dosage. An inhibitory effect on the endothelial cell anticoagulant function may also have consequences during hypothermic low flow and circulatory arrest states. ${ }^{5}$ Thus many early patients were inadequately anticoagulated at stages of their operation.

With generous financial support from the Bayer Corporation (Leverkusen, Germany), a number of clinical and scientific studies showed the hemostatic mechanism to result from protection of platelet adhesive receptors at the onset of cardiopulmonary bypass. ${ }^{6}$ Without aprotinin, the contact system of plasma is massively activated on first passage through the oxygenator. Activation of the intrinsic coagulation pathway causes thrombin formation, which impairs platelet adhesive function. Aprotinin also blocks the contact activation of the kallikrein system and, in synergy with heparin, prevents thrombin formation through inhibition of the intrinsic clotting cascade. ${ }^{7}$ Although this is not the whole story, it is likely that neither thrombin nor platelets become involved in the blood-foreign surface contact activation process in aprotinin-treated patients. The fact that the hemostatic process is affected from the very beginning of cardiopulmonary bypass is substantiated by the fact that low-dose aprotinin $\left(2 \times 10^{6} \mathrm{KIU}\right)$ added to the pump prime leads to the same preservation effect on platelet receptors and blood loss as continuous high-dose infusion $\left(6 \times 10^{6} \mathrm{KIU}\right)$ throughout the procedure. This observation had clinical implications inasmuch as 


\section{Abbreviations and Acronyms}

ACT = activated clotting time

BART $=$ Blood Conservation using Antifibrinolytics:

A Randomized Trial in High-Risk Cardiac

Surgery Patients

$\mathrm{CABG}=$ coronary artery bypass grafting

FDA $=$ Food and Drug Administration

HCA = hypothermic circulatory arrest

a single pump prime dose of aprotinin was much less expensive than the high-dose "Hammersmith regimen."

Aprotinin became commercially available and enjoyed widespread use in Europe before these mechanisms were fully elucidated. First licensed for use in patients at high risk of postoperative bleeding (reoperations, endocarditis, multiple valve replacements), the indications were soon extended to the commercially important realm of coronary bypass surgery (CABG) in patients taking aspirin. Extensive aortic surgery provided an obvious role for aprotinin, but even after the target celite ACT was raised to greater than 700 seconds the anticoagulation of completely static blood during hypothermic circulatory arrest would prove problematic. Cooling alone prolongs the ACT, so higher doses of heparin are needed when profound hypothermia is used (ACT $>1000$ seconds). ${ }^{8}$ During hypothermic circulatory arrest (HCA), the endothelial cell normally produces substantial amounts of tissue plasminogin activator, which produces plasmin and activates fibrinolysis to prevent vascular occlusion by fibrin. Thrombin formation during stagnation also stimulates the protein $\mathrm{C}$ system of the endothelial lining. This inhibits the actived clotting factors Va and VIIIa to minimize coagulation. However, both plasmin and protein $\mathrm{C}$ are inhibited by aprotinin. Fatal intravascular coagulation and renal failure were encountered in early series, sparking vigorous debate over safety and adequacy of anticoagulation after HCA. ${ }^{9,10}$

European observational and randomized studies all confirmed the hemostatic effect, the reduction in blood transfusion requirement, and less frequent return to the operating theater to control bleeding. ${ }^{11}$ Many European centers gave aprotinin to every patient undergoing cardiac surgery. Different dosage regimens were tried and most found to be effective in reducing blood loss. Aprotinin was even used as a topical agent in the pericardium and for postoperative infusion during established bleeding. Few if any of the randomized studies were powered to show differences in mortality.

\section{Food and Drug Administration (FDA) Approval and Beyond}

Aprotinin had no license for any indication in the United States and further studies were required with varied dose regimens before FDA approval. Because these trials were primarily designed to show effectiveness, they were also individually too small to establish safety. As expected, the hemostatic effect was confirmed with a reduction in transfusion requirement for so-called higher-risk patients, but there was no significant benefit for CABG in patients without antiplatelet therapy. ${ }^{12}$ Two trials in patients requiring reoperative CABG reported excess mortality in the half-dose arm and increased myocardial infarction rate by laboratory criteria. ${ }^{13,14}$ Cosgrove and colleagues ${ }^{13}$ raised concern over early graft thrombosis and mortality based on autopsy findings but with a trend that failed to meet statistical significance. Levy and associates ${ }^{14}$ noted increased mortality and myocardial infarction with a half-dose regimen, but the study was underpowered for mortality $(P=.354)$. Lemmer and colleagues ${ }^{12}$ showed a significantly increased rate of myocardial infarction by laboratory criteria (without elevated mortality) when only the pump prime dose was used. In contrast, the full-dose regimen advocated by Bayer did not elevate risk of mortality or ischemic events, and many interpreted the results as demonstrating aprotinin to be safe and effective for all patients.

Because of the persisting uncertainty surrounding adverse events, Smith and Muhlbaier ${ }^{15}$ pooled all the raw data from the four published US trials together with two other unpublished studies obtained from the Bayer Corporation. This allowed more powerful analysis of myocardial infarction, stroke, renal failure, and mortality. The analysis showed CABG mortality for half-dose aprotinin to be $5.4 \%$ compared with $3.8 \%$ for placebo (a $42 \%$ increase), yet the number of randomized patients (2283) was still too small to detect statistically significant differences. Power analysis shows that 3160 patients would be required for each group to detect a $40 \%$ increase in mortality (from $3.8 \%$ to $5.3 \%$ ). Again, there was no difference in mortality in the larger cohort randomized to high-dose aprotinin or placebo $(2.7 \%$ vs $2.8 \%)$. No difference was noted in renal dysfunction, but there was an intriguing reduction in stroke rate for patients receiving a full-dose regimen (1.0\% vs $2.4 \%$ placebo; $P=.27$ ).

Royston, ${ }^{16}$ a coworker for the original Hammersmith study, provided an elegant pathophysiologic hypothesis to explain how low-dose therapy might cause a greater thrombotic risk than high dosage $\left(6 \times 10^{6} \mathrm{KIU}\right)$. Aprotinin at high dose inhibits both coagulation (resulting in reduced thrombin formation) and fibrinolysis (causing less D-dimer formation). At lower doses, the anticoagulant function mediated through kallikrein inhibition is less prominent. The plasma concentration recognized to inhibit kallikrein is 200 $\mathrm{KIU} / \mathrm{mL}$ or greater whereas the plasma inhibition level is only $50 \mathrm{KIU} / \mathrm{mL}$. Accordingly the "Hammersmith" dose aprotinin regimen combines both anticoagulant and antifibrinolytic effects as well as preservation of platelet function. In contrast, low plasma concentrations maintain fibrinolysis inhibition but lose inhibition of the intrinsic clotting cascade. This state may predispose to the thrombotic complications described. ${ }^{12}$ 
So that the safety of aprotinin in patients undergoing CABG could be established, there was a need for invasive studies of coronary graft patency. Again, these produced conflicting information depending on the dosage regimen and mode of investigation. Most investigators using early graft angiography or ultrafast computed tomography suggested no difference between placebo and treatment groups. ${ }^{17}$ In contrast, a nonrandomized retrospective study by van der Meer and colleagues ${ }^{18}$ using graft angiography 1 year after CABG showed distal anastomosis occlusion rates of $20.5 \%$ for treated patients versus $12.7 \%$ without aprotinin $(P=.09)$. The proportion of patients with occluded versus patent grafts was $44.1 \%$ versus $26.3 \%$, respectively $(P=.3)$. Perioperative myocardial infarction occurred in $14.3 \%$ versus $7.0 \%(P=.12)$. Bayer then commissioned a multicenter trial using graft angiography to be performed within a few days of surgery. ${ }^{19}$ Patients were recruited from 10 centers in the United States, 2 in Israel, and 1 in Denmark (designated "European centers"). During 1994 and 1995, 870 patients were randomized to either high-dose aprotinin or placebo. The outcome was eventually reported 2 years later at the 78th Annual Meeting of The American Association for Thoracic Surgery (1998). Only $703(80 \%)$ patients had satisfactory graft angiograms, which showed thrombotic occlusion in $15.4 \%$ of aprotinintreated patients versus $10.9 \%$ in the placebo group $(P=$ .03). However, 1 non-US center showed an unexpectedly high graft occlusion rate; when analysis of graft closures was repeated for US centers only, there was no significant difference between aprotinin and placebo.

Why were the differences in vein graft occlusion rates between the US and non-US centers so dramatic (aprotinin; non-US 23\%, US 9.4\%: placebo; non-US $12.4 \%$, US $9.5 \%)$ ? Scrutiny of the data provides several explanations. The study protocol was changed at the Israeli site after blood for vein preparation was initially obtained from the central venous line into which aprotinin had been administered, and this produced a graft occlusion rate of $73 \%$ in the aprotinin group and an important indication of the risk of this agent in patients with coronary disease. After procedures at this center were revised, graft occlusion rates with aprotinin decreased to $14 \%$ versus $3 \%$ for placebo for the remaining two thirds of the study. There were other important differences. First, the patients were selected, not consecutive, and the coronary surgery intervention rates in the United States $\left(>1000 \times 10^{6}\right)$ greatly exceeded those of European centers $\left(<500 \times 10^{6}\right)$. Despite this, the US centers on average randomized only 47 patients over 2 years, of whom $90 \%$ were male. One center randomized only 2 patients. Patients in US centers were well documented to have larger and better quality distal vessels. US sites also had a higher proportion of patients receiving aspirin within 2 days of the operation, which would favor graft patency. Consequently, the US patient cohort was less likely to have graft occlusion with or without aprotinin. In contrast, European centers randomized on average 133 patients per center, more women, more patients with vessels less than $1.5 \mathrm{~mm}$ in diameter, and more with distal disease. These variables were subsequently identified as predisposing to graft occlusion in aprotinintreated patients.

Given the status of interventional cardiology in 2008, the patients randomized in the non-US centers now clearly represent the global CABG population and could be interpreted as "at risk" from aprotinin during CABG. Occluded grafts are a high price to pay for an average blood saving of $250 \mathrm{~mL}$, particularly inasmuch as pump prime volume, as well as blood loss, determines the postoperative hematocrit in most straightforward operations. ${ }^{12}$ There are other methods to conserve blood, and the contrary view that hemodilution, temporary fibrinolysis, and platelet dysfunction promote vein graft patency is more appealing.

Claims and counterclaims over safety issues have continued over the 15 years since FDA approval in 1993. Very few trials have been powered to detect increased mortality or myocardial infarction rates, and because of the cost of aprotinin, most studies have been sponsored and policed by the manufacturers. This key issue was identified as a source of bias by Mangano, Tudor, and Dietzel ${ }^{20}$ on publication of their manuscript, "The Risk Associated With Aprotinin in Cardiac Surgery." The hypothesis underpinning the Mangano study challenged the logic of aprotinin use in patients with acute coronary syndrome undergoing CABG who should otherwise be subject to the contrary strategies of platelet inhibition and fibrinolysis. This observational study of patients undergoing CABG by the Multicentre Study of Perioperative Ischaemia Research Group assessed the 3 antifibrinolytic agents-aprotinin (1295 patients), aminocaproic acid (883 patients), and tranexamic acid (822 patients) — as compared with no agent (1374 patients). A propensityadjusted multivariate logistic regression analysis showed aprotinin to be associated with a doubling of the risk of renal failure requiring dialysis, a 55\% increase in the risk of myocardial infarction or heart failure $(P=.001)$, and a $181 \%$ increase in stroke or encephalopathy $(P=.001)$. The other agents were blameless in this respect. The authors stated the obvious by suggesting that the combination of kallikrein, plasmin, and protein $\mathrm{C}$ inhibition, together with preservation of platelet function and impairment of vasculoendothelial function, provided a convincing substrate for the observed thrombotic effects.

True to form, Mangano's paper was vigorously attacked. Royston, van Haaften, and de Vooght ${ }^{21}$ provided a scholarly rebuttal of virtually all studies that reported adverse events and ended emotionally with the statement "If the NEJM article is incorrect and patients suffer increased transfusions leading to renal failure and death because aprotinin therapy was withheld due to fear of litigation what debt is owed to our patients for such information? 
Furnary and colleagues ${ }^{22}$ used a nonrandomized retrospective observational study to contest the recurring allegation that aprotinin predisposes to renal failure. They suggested that aprotinin had been used in selected higherrisk patients and that it was an increase of transfused packed red blood cells that independently caused postoperative acute renal failure, not aprotinin itself. Sedrakyan, Atkins, and Treasure $^{23}$ chose the Lancet to dispute Mangano's findings. They implied that the clinical decision to use aprotinin in high-risk patients (the way the agent was meant to be used) caused large differences between patient groups and that differential exclusion of patients added bias. In fact, the majority of Mangano's excluded patients had received lowdose therapy, previously identified to elevate risk, and had $7.2 \%$ mortality compared with $2.6 \%$ mortality among included patients. Sedrakyan, Treasure, and Elefteriades ${ }^{24}$ argued that their own meta-analysis of 35 randomized but grossly underpowered trials of CABG patients (little more than 100 patients per trial) indicated that there was no increased risk of myocardial infarction, renal failure, or stroke. These authors suggested the need for a new appropriately powered prospective randomized trial to compare aprotinin with the other antifibrinolytics and signed off with the now resounding words, "We await the findings of the BART trial in Canada."

\section{Fallout From the BART Trial}

BART stands for "Blood Conservation using Antifibrinolytics: A Randomized Trial in High-Risk Cardiac Surgery Patients." The study was designed to test the hypothesis that aprotinin was superior to epsilon-aminocaproic acid or tranexamic acid in decreasing the occurrence of major bleeding in cardiac surgery. Described as an investigatorsponsored independent randomized controlled trial, the study intended to enroll around 3000 adult patients in Canada who fulfilled the criteria for elevated risk of postoperative bleeding. However, on October 19, 2007, the Data Safety Monitoring Board informed the FDA that at an interim analysis the 30-day mortality in the aprotinin group had already virtually reached conventional statistical significance when compared with the other agents. A trend toward higher mortality had been apparent for the aprotinin group throughout the study (relative risk of 1.5 compared with the other agents). Paradoxically, more deaths from hemorrhage were recorded in aprotinin-treated patients. The Data Safety Monitoring Board considered that further recruitment to the study was unlikely to change these findings, and the FDA took the view that BART reinforced the findings of Mangano and others who had reported increased risk of mortality with aprotinin use. Although the FDA did not place an outright ban on aprotinin, it issued a warning and asked for reports of serious or unexpected adverse reactions. After this review, Bayer and Nordic Pharma (Baarn, The Netherlands) voluntarily suspended global marketing of the drug. The
Commission for Human Medicines suspended relevant United Kingdom licenses because specific patient groups at increased risk of mortality or those who may gain most benefit from aprotinin could not be identified on current information. There will now be a full risk-benefit review by authorities in Europe and the United States.

Clinical trials involving "miscellaneous high-risk patients" are seldom straightforward, but this is the real world of antifibrinolytic therapy in cardiac surgery. It is inevitable that the BART study will be subject to the same battery of criticism. So far, the causes of death have not been presented in detail, but excess bleeding in aprotinin-treated patients was a feature of some HCA case studies and followed inadequate heparinization and disseminated intravascular coagulation. ${ }^{9}$ Greater transfusion requirement was noted for aprotinintreated patients in a retrospective study of aprotinin, blood transfusion, and renal impairment by Furnary and associates. ${ }^{22}$ Whether other deaths were related to thrombotic or bleeding events remains to be seen, but Royston's realization $^{21}$ of the potential for litigation after any adverse event in an aprotinin-treated patient must now be taken seriously. Recently, in this Journal Augoustides ${ }^{25}$ an anesthesiologist, described fatal widespread thrombosis in 5 patients after HCA operations in which aprotinin or epsilon-aminocaproic acid had been used. He was convinced that standard of care anticoagulation had been maintained throughout, but factor $\mathrm{V}$ Leiden deficiency was identified in 2 of the 5 patients. This genetically based hypercoaguable condition is found in around $8 \%$ of cardiac surgical patients and aprotinin may contribute to thrombosis risk by inhibition of protein C. Most cardiac centers do not screen for factor V Leiden deficiency, thus adding an unpredictable factor to antifibrinolytic therapy in a significant proportion of patients.

\section{My Perspective}

No drug can be regarded as completely safe, and patient variability plays an important role in susceptibility to adverse events. Aprotinin is used in the full age spectrum from neonatal to geriatric cardiac surgery. Since clinical trials powered to detect adverse events emerged only after 20 years of marketing, it is probably fair to conclude that commercialization forged ahead of science. A risk-benefit analysis must now cover all age groups and clinical conditions.

I have used aprotinin selectively since initiating the Hammersmith trial in 1982. In more than 10,000 operations with cardiopulmonary bypass, aprotinin was used in approximately 3500 patients. These all fell into so-called high-risk groups, including early (not late) reoperation, surgery for complex congenital heart defects, multiple (but rarely single) valve replacements, mechanical circulatory support, endocarditis, and in thoracic aortic aneurysm surgery. The latter includes patients with acute type A dissection after initially reporting a series of adverse events, which were successfully addressed by changing anticoagulation policy. ${ }^{9}$ My team and 
I have avoided aprotinin in all patients undergoing CABG unless subject to recent thrombolysis or clopidogrel therapy within 48 hours of surgery. We have always looked for drug-related adverse events and performed autopsies after postoperative death. Since 1990 only one death with multiple thrombotic events and clotting in the oxygenator occurred that could have been considered aprotinin related, and in retrospect that patient probably had factor V Leiden deficiency. There have been 4 easily controlled anaphylactic events in response to a test dose of aprotinin. At the same time, we have achieved less than $1 \%$ mortality for elective aortic root operations, $2 \%$ mortality for complete atrioventricular valve defects in infancy, and 6\% mortality for acute type A dissection. These are patient groups in which aprotinin has been used routinely for many years. With very experienced anesthesiologists and use of the Hammersmith dosage regimen, we consider the risk-benefit equation to strongly favor aprotinin use in the type of patient described. We regard aprotinin as very safe when used selectively with close attention to anticoagulation management and feel profoundly disadvantaged to be left without a supply.

\section{References}

1. Despotis G, Avidan MS, Hogue CW. Mechanisms of hemostatic activation during extracorporeal circulation. Ann Thorac Surg. 2001;72: 1821-31.

2. Koch CG, Li L, Duncan AL, Mihaljevic T, Loop FD, Starr NJ, et al. Transfusion in coronary artery bypass grafting is associated with reduced long-term survival. Ann Thorac Surg. 2006;81:1650-7.

3. Kuduvalli M, Oo AY, Newall N, Grayson AD, Jackson M, Desmond MJ, et al. Effect of peri-operative red blood cell transfusion on 30-day and 1-year mortality following coronary artery bypass surgery. Eur J Cardiothorac Surg. 2005;27:592-8.

4. de Smet AA, Joen MC, van Oeveren W, Roozendaal KJ, Harder MP, Eijsman L, et al. Increased anticoagulation during cardiopulmonary bypass by aprotinin. J Thorac Cardiovasc Surg. 1990;100:520-7.

5. Westaby S. Coagulation disturbances in profound hypothermia. Semin Thorac Cardiovasc Surg. 1997;9:246-56.

6. Wildevuur CR, Eijsman L, Roozendaal KJ, Harder MP, Chang M, van Oeveren W, et al. Platelet preservation during cardiopulmonary bypass with aprotinin. Eur J Cardiothorac Surg. 1989;3:533-8.

7. Westaby S. Aprotinin in perspective. Ann Thorac Surg. 1993;55: 1033-41.

8. Okita $\mathrm{Y}$, Takamoto $\mathrm{S}$, Ando $\mathrm{M}$, et al. Coagulation in fibrinolysis system in aortic surgery under deep hypotheric circulatory arrest with aprotinin. Circulation. 1997;96(9 Suppl): 11-376-81.

9. Westaby S, Forni A, Dunning J, Giannopoulos N, O'Regan D, Drossos G, et al. Aprotinin and bleeding in profoundly hypothermic perfusion. Eur J Cardiothoracic Surg. 1994;8:82-6.
10. Sundt TM 3rd, Saffitz JE, Stahl J, Murphy SF, Wareing TH, Stahl DJ. Renal dysfunction and intravascular coagulation after use of aprotinin in thoracic aortic operation employing hypothermic circulatory arrest. Ann Thorac Surg. 1993;55:1418-24.

11. Royston D, Bidstrup BP, Taylor KM, Sapsford RN. Effect of aprotinin on need for blood transfusion after repeat open heart surgery. Lancet. 1987;2:1289-91.

12. Lemmer JH Jr, Dilling EW, Morton JR, Rich JB, Robicsek F, Bricker DL, et al. Aprotinin for primary coronary artery bypass grafting: a multicenter trial of three dose regimens. Ann Thorac Surg. 1996;62: 1659-67.

13. Cosgrove DM 3rd, Heric B, Lytle BW, Taylor PC, Novoa R, Golding LA, et al. Aprotinin therapy for reoperative myocardial revascularization: a placebo-controlled study. Ann Thorac Surg. 1992;54: 1031-6.

14. Levy JH, Pifarré R, Schaff HV, Breen JF, Chomka EV, Eldredge WJ, et al. A multicenter, double-blind, placebo-controlled trial of aprotinin for reducing blood loss and the requirement for donor-blood transfusion in patients undergoing repeat coronary artery bypass grafting. $J$ Thorac Cardiovasc Surg. 1994;107:543-51.

15. Smith PK, Muhlbaier LH. Aprotinin: safe and effective only with the full-dose regimen. Ann Thorac Surg. 1996;62:1575-7.

16. Royston D. Intraoperative coronary thrombosis: can aprotinin be incriminated. J Cardiothorac Vasc Anesth. 1994;8:137-41.

17. Havel M, Grabenwoger F, Schneider J, Laufer G, Wollenek G, Owen A, et al. Aprotinin does not decrease early graft patency after coronary bypass grafting despite reducing postoperative bleeding and use of donated blood. J Thorac Cardiovasc Surg. 1994;107:807-10.

18. van der Meer J, Hillege HL, Ascoop CA, Dunselman PH, Mulder BJ, van Ommen GV, et al. Aprotinin in aorto-coronary bypass surgery: increased risk of vein graft occlusion and myocardial infarction? Supportive evidence from a retrospective study. Thromb Haemost. 1996;75:1-3.

19. Alderman EL, Levy HJ, Rich JB, Nili M, Vidne B, Schaff H, et al. Analyses of coronary graft patency after aprotinin use: results from the International Multicenter Aprotinin Graft Patency Experience (IMAGE) trial. J Thorac Cardiovasc Surg. 1998;116:716-30.

20. Mangano DT, Tudor IC, Dietzel C. The risk associated with aprotinin in cardiac surgery. $N$ Engl J Med. 2006;354:353-65.

21. Royston D, van Haaften N, de Vooght P. Aprotinin: friend or foe? A review of recent medical literature. Eur J Anaesthesiol. 2007;24:6-14.

22. Furnary AP, Wu YX, Hiratzka LF, Grunkemeier GL, Page US 3rd. Aprotinin does not increase the risk of renal failure in cardiac surgery patients. Circulation. 2007;116(11 Suppl I):I127-33.

23. Sedrakyan A, Atkins D, Treasure T. The risk of aprotinin: a conflict of evidence. Lancet. 2006;367:1376-7.

24. Sedrakyan A, Treasure T, Elefteriades JA. Effect of aprotinin on clinical outcomes in coronary artery bypass surgery: a systematic review and meta analysis of randomized clinical trials. J Thorac Cardiovasc Surg. 2004; 128:442-8.

25. Augoustides JGT. Fatal intraoperative thrombosis in contemporary adult thoracic aortic surgery requiring deep hypothermic circulatory arrest: observations from the literature 1993-2006. J Thorac Cardiovasc Surg. 2007;134:1069-70. 